\title{
Protective Effect of Adipose-Derived Mesenchymal Stem Cell Secretome against Hepatocyte Apoptosis Induced by Liver Ischemia-Reperfusion with Partial Hepatectomy Injury
}

\author{
Zhihui Jiao $\mathbb{D}^{1,2}$ Yajun Ma $\mathbb{D}^{1,}{ }^{1}$ Yue Wang, ${ }^{1}$ Tao Liu, ${ }^{1}$ Qianzhen Zhang, ${ }^{1}$ Xiaoning Liu, \\ Chenxi Piao $₫{ }^{1},{ }^{1}$ Boyang Liu, ${ }^{1,2}$ and Hongbin Wang ${ }^{1}$ \\ ${ }^{1}$ College of Veterinary Medicine, Northeast Agricultural University, Harbin, China \\ ${ }^{2}$ College of Wildlife and Protected Area, Northeast Forestry University, Harbin, China \\ Correspondence should be addressed to Hongbin Wang; hbwang1940@neau.edu.cn
}

Received 11 March 2021; Revised 7 July 2021; Accepted 29 July 2021; Published 19 August 2021

Academic Editor: Stefan Arnhold

Copyright (c) 2021 Zhihui Jiao et al. This is an open access article distributed under the Creative Commons Attribution License, which permits unrestricted use, distribution, and reproduction in any medium, provided the original work is properly cited.

Ischemia-reperfusion injury (IRI) is an inevitable complication of liver surgery and liver transplantation. Hepatocyte apoptosis plays a significant role in the pathological process of hepatic IRI. Adipose-derived stem cells (ADSCs) are known to repair and regenerate damaged tissues by producing bioactive factors, including cytokines, exosomes, and extracellular matrix components, which collectively form the secretome of these cells. The aim of this study was to assess the protective effects of the ADSCs secretome after liver ischemia-reperfusion combined with partial hepatectomy in miniature pigs. We successfully established laparoscopic liver ischemia-reperfusion with partial hepatectomy in miniature pigs and injected saline, DMEM, ADSCsecretome, and ADSCs directly into the liver parenchyma immediately afterwards. Both ADSCs and the ADSC-secretome improved the IR-induced ultrastructural changes in hepatocytes and significantly decreased the proportion of TUNEL-positive apoptotic cells along with caspase activity. Consistent with this, P53, Bax, Fas, and Fasl mRNA and protein levels were markedly decreased, while Bcl-2 was significantly increased in the animals treated with ADSCs and ADSC-secretome. Our findings indicate that ADSCs exert therapeutic effects in a paracrine manner through their secretome, which can be a viable alternative to cell-based regenerative therapies.

\section{Introduction}

Hepatic ischemia-reperfusion injury (HIRI), a precursor to liver dysfunction and liver failure [1], is an inevitable complication of shock, trauma, hepatectomy, liver transplantation, and other surgical procedures [2-4]. The pathophysiological process of HIRI involves excessive production of reactive oxygen species (ROS), activation of Kupffer cells and other inflammatory cells, and calcium overload, which eventually lead to hepatocellular apoptosis [5]. Although orthotopic liver transplantation (OLT) is an effective treatment for terminal liver dysfunction, it is limited by organ shortage, high costs, immune rejection, and transplant-related complications [6]. HIRI is still an unresolved clinical issue, and an effective strategy is urgently needed to alleviate HIRI and improve patient prognosis.
Stem cell therapy is a promising approach for tissue repair and regeneration [7-9]. Mesenchymal stem cells (MSCs) in particular have shown encouraging results against inflammatory, degenerative, and ischemia-reperfusion diseases [10-12] since they can be isolated from multiple sources, including adipose tissue [13], bone marrow, dental pulp [14], umbilical cord blood [15], tonsils [16], oral cavity [17], and amniotic fluid [18]. Adipose-derived stem cells (ADSCs) are increasingly being considered a promising tool for cellular therapy and tissue engineering [19]. Depending on the environmental stimuli, ADSCs can differentiate into osteoblasts, adipocytes, and hepatocytes [20] and are therefore highly suitable for cell-based therapy in multiple organ systems. However, the clinical application of stem cells is limited by long-term safety concerns, such as unwanted differentiation [21], potential tumorigenicity [22], and elimination 
by the receptor immune system [23]. Therefore, stem cell transplantation is still at the experimental stage [24].

The regenerative effects of transplanted stem cells are mainly attributed to the paracrine regulation of endogenous cells via secreted factors [25]. The secretome of a cell population refers to the biologically active factors secreted by the cells into the extracellular space, including soluble proteins, free nucleic acids, lipids, and extracellular vesicles [26], that aid in intercellular communication and transport. Several studies have demonstrated the regenerative potential of the stem cell secretome, which can obviate some of the pressing concerns of cell-based therapies, including immune rejection, tumorigenicity, and emboli formation.

Adipose-derived stem cell conditioned medium (ADSC$\mathrm{CM}$ ) or secretome has shown remarkable therapeutic effects in small-animal models of angiogenesis [27], diabetic pain [28], wound healing [29], glucose metabolism [30], etc. While stem cell therapy has been investigated in animal models of partial hepatectomy, little is known regarding the effect of ADSC-secretome on HIRI in large animals. In this study, we established laparoscopic hepatic ischemiareperfusion and partial hepatectomy in miniature pigs and transplanted ADSCs or the ADSC-secretome directly into the liver parenchyma. The ADSC-secretome alleviated apoptosis in the hepatocytes and improved cellular ultrastructure. Our findings show that the ADSC-secretome is a safe and effective strategy against HIRI.

\section{Materials and Methods}

2.1. ADSC Culture and Preparation of Conditioned Medium $(C M)$. Adipose tissues were obtained from the subcutaneous abdominal fat and digested with collagenase I at $37^{\circ} \mathrm{C}$ for 45 min with continuous shaking. After neutralizing enzyme activity with L-DMEM (low glucose-Dulbecco's modified Eagle medium) supplemented with 10\% FBS (Clark, USA), the homogenate was filtered and centrifuged, and the ADSCs were suspended in L-DMEM supplemented with 10\% FBS, $2 \mathrm{mM}$ L-glutamine, and $100 \mu \mathrm{g} / \mathrm{ml}$ penicillin and streptomycin (Solarbio, China). The cells were cultured at $37^{\circ} \mathrm{C}$ under $5 \% \mathrm{CO}_{2}$ in a humidified incubator (Galaxy $170 \mathrm{~S}$, Eppendorf, Germany). The ADSCs were characterized as previously described [31], cultured in serum-free L-DMEM for $48 \mathrm{~h}$. Then, the medium was aspirated and centrifuged at $1000 \mathrm{~g}$ for $15 \mathrm{~min}$ at $4^{\circ} \mathrm{C}$ to remove the cell debris. The supernatant was then centrifuged at $5000 \mathrm{~g}$ for $50 \mathrm{~min}$ at $4^{\circ} \mathrm{C}$ using $3 \mathrm{kDa}$ MWCO (Millipore, Billerica, USA) to concentrate it by 25 -fold. The $\mathrm{CM}$ aliquots were transferred to sterile $1.5 \mathrm{ml} \mathrm{EP}$ tubes and stored at $-80^{\circ} \mathrm{C}$.

2.2. Surgical Procedure. Twenty-four miniature pigs (age: 4-6 months, body weight: $20-25 \mathrm{~kg}$ ) were provided by the Miniature Pig Farm of the College of Life Sciences (Harbin, China). The animals were housed at $20^{\circ} \mathrm{C}$ under a $12 \mathrm{~h}$ light-dark cycle and fed piglet diet (Shenzhen Jinxinnong Feed, China) and tap water ad libitum. The animals were randomly divided into the (untreated) IRI, DMEM control, CM, and ADSC groups ( $n=6$ per group). After $12 \mathrm{~h}$ fasting and $2 \mathrm{~h}$ water deprivation, the animals were anesthetized with isoflurane inhalation and subjected to laparoscopic left hepatectomy after right hepatic ischemia for $60 \mathrm{~min}$. Immediately after the operation, the liver parenchyma was injected with saline (IRI group), DMEM, ADSCs $\left(1 \times 10^{6}\right.$ $\mathrm{P} 4$ cells/kg body weight) or ADSC-secretome (CM equivalent to $1 \times 10^{6} \mathrm{P} 4 \mathrm{ADSCs} / \mathrm{kg}$ ). The animals were euthanized by injecting $4 \%$ tolfedine (Vetoquinol S.A., France). Liver tissues were laparoscopically harvested preoperation and 1,3 , and 7 days postoperation. All the animals survived during the entire duration of the experiment due to the minimally invasive surgery.

2.3. Transmission Electron Microscopy. The liver samples were cut into $1 \mathrm{~mm}^{3}$ pieces and fixed with $2.5 \%$ glutaraldehyde. After routine dehydration, the tissues were embedded, sectioned, and stained with lead citrate and uranyl acetate. The ultrastructural changes in the hepatocytes were observed using an H-7650 transmission electron microscope (Hitachi, Japan).

2.4. TUNEL Analysis. The liver tissues were fixed with $4 \%$ paraformaldehyde ( $n=6$ per group), embedded with paraffin, and cut into sections. After dewaxing and dehydrating, the sections were stained with TUNEL assay using an In Situ Cell Death Detection Kit (Roche, Germany) according to the manufacturer's instructions. The TUNEL-positive cells were counted in five random fields of each section, and its percentage relative to the total number of hepatocytes was calculated.

2.5. Caspase Activity Analysis. Liver tissues were homogenized with lysis buffer ( $n=6$ per group), and the activities of caspase 3, caspase 8 , and caspase 9 were determined using specific Caspase Activity Assay Kits (Solarbio, China) according to the manufacturer's instructions.

2.6. Real-Time Quantitative PCR Analysis. Total RNA was extracted from the liver tissues using a TRIzol reagent (Invitrogen, Shanghai, China) according to the manufacturer's instructions. Reverse transcription was performed using a PrimeScript $^{\mathrm{TM}}$ RT Reagent Kit (Takara, Japan). RT-qPCR was performed using the SYBR Green Kit in a LightCycler 480 System (Roche Applied Science, Penzberg, Germany) with the following cycling parameters: predenaturation at $95^{\circ} \mathrm{C}$ for $30 \mathrm{~s}$, followed by 40 cycles of denaturation at $95^{\circ} \mathrm{C}$ for $5 \mathrm{~s}$, and annealing and elongation at $60^{\circ} \mathrm{C}$ for $1 \mathrm{~min}$. The threshold cycle (CT) values were calculated by the $2^{-\Delta \Delta \mathrm{Ct}}$ method (Livak and Schmittgen, 2001). The primers are listed in Table 1.

2.7. Western Blotting. Liver tissues were homogenized using a Tissue Protein Extraction Reagent (Beyotime, Shanghai, China) for $30 \mathrm{~min}$ at $4^{\circ} \mathrm{C}$. The homogenates were centrifuged at $12,000 \mathrm{~g}$ for $15 \mathrm{~min}$ at $4^{\circ} \mathrm{C}$, and the protein concentration was determined using a Bicinchoninic Acid (BCA) Protein Assay Kit (Beyotime, Shanghai, China). Equal amounts of protein were separated by sodium dodecyl sulfate-polyacrylamide gel electrophoresis (SDS-PAGE) and then transferred onto nitrocellulose (NC) membranes. After blocking with 5\% nonfat milk in TBST for $2 \mathrm{~h}$ at room temperature, the membranes were incubated overnight with anti-P53, anti-Bcl-2 (Wanlei 
TABLE 1: Sequences of primers used in real-time PCR.

\begin{tabular}{lrr}
\hline Gene & Forward primer $\left(5^{\prime}->3^{\prime}\right)$ & Reverse primer $\left(5^{\prime}->3^{\prime}\right)$ \\
\hline P53 & CCTCACCATCATCACACTGG & TTGGCCCTTCTTGAGGAAAT \\
Bax & TTCAGGGTTTCATCCAGGATCG & ATCCTCTGCAGCTCCATGTTAC \\
Bcl-2 & GAGGATTGTGGCCTTCTTTG & GCCGGTTCAGGTACTCAGTC \\
Fas & TGTCCGGGATCTGGGTTCTC & GGCATGGCTGACAGCAGAAT \\
Fasl & ACCACCACCACTCCTGCCATC & TCCCCAGCCCCAATCCAACC \\
$\beta$-Actin & TCTGGCACCACACCTTCT & TGATCTGGGTCATCTTCTCAC \\
\hline
\end{tabular}
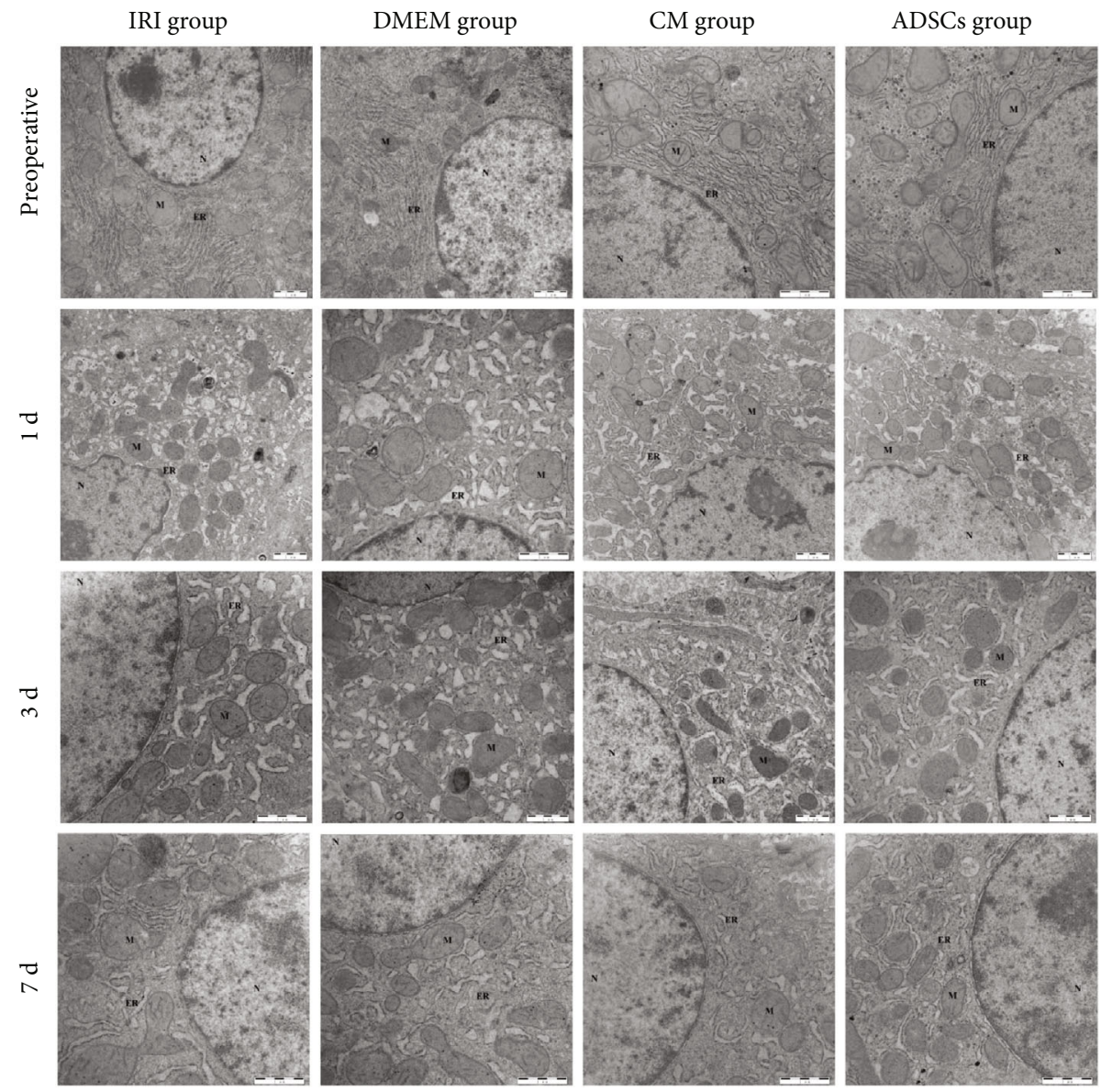

FIgURE 1: The ultrastructural changes in hepatocytes.

Biology, Shenyang, China), anti-Bax, and anti- $\beta$-actin (Sangon Biotech, Shanghai, China) primary antibodies. The membranes were then washed with TBST and incubated with horseradish peroxidase-conjugated secondary antibody (ImmunoWay, Plano, USA) at room temperature for $2 \mathrm{~h}$. Following another wash with TBST, the blots were developed using a Meilunbio ${ }^{\circledR}$ fg super-sensitive ECL luminescence reagent (Meilunbio, Dalian, China) and imaged using the Tanon 5200 Imaging System (Tanon Science \& Technology Co., Shanghai, China). The relative density of the target bands was quantified using ImageJ software.

2.8. Immunohistochemistry. Paraffin-embedded tissue sections were deparaffinized, dehydrated, and treated with $3 \%$ hydrogen peroxide in the dark for $10 \mathrm{~min}$ to inactivate the endogenous peroxidases. After heating in $0.01 \mathrm{M}$ citrate buffer in the microwave for $10 \mathrm{~min}$ for antigen retrieval, the sections were cooled to room temperature, blocked with bovine serum albumin (BSA), and incubated overnight with anti-Fas and anti-Fasl primary antibodies (ImmunoWay, Plano, USA) at $4^{\circ} \mathrm{C}$. The sections were then incubated with a streptavidin-labeled HRP secondary antibody (ZSGB, Beijing, China) for $30 \mathrm{~min}$ at room temperature, followed by a $\mathrm{DAB}$ solution for $3 \mathrm{~min}$. After counterstaining with hematoxylin, the positively stained sections were quantified with the Image-Pro Plus 6.0 software (Media Cybernetics, USA). Six tissue sections per group and five random fields per slide were analyzed. 

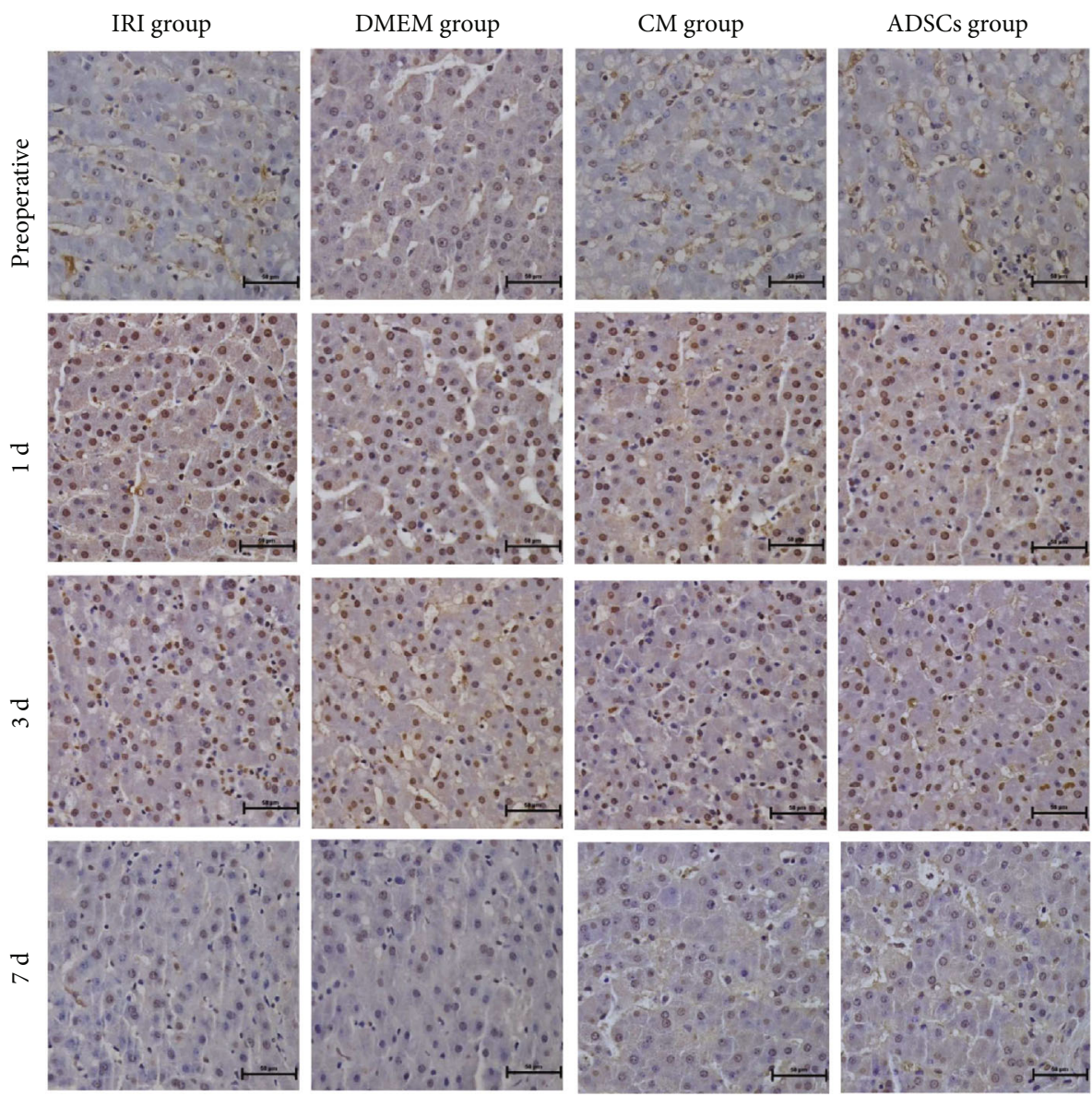

(a)

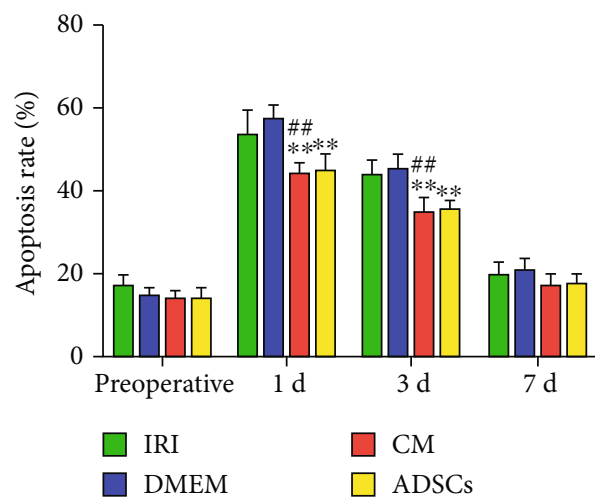

(b)

FIGURE 2: Hepatocyte apoptosis rates: (a) representative images of TUNEL-stained liver tissues from the indicated groups and at the indicated time points (magnification $400 \mathrm{x}$ ); (b) apoptosis rate of hepatocytes. ${ }^{* *} P<0.01$ compared to IRI group and ${ }^{\# \#} P<0.01$ compared to DMEM group.

2.9. Statistical Analysis. All data were analyzed with GraphPad Prism 7.0 (GraphPad Software, USA) and expressed as mean \pm SD. One-way ANOVA was used to compare different groups, and $P<0.05$ was considered statistically significant.

\section{Results}

3.1. ADSCs/ADSC-Secretome Relieved the Ultrastructural Damage in Injured Hepatocytes. As shown in the electron micrographs in Figure 1, the nuclei, mitochondria, and endoplasmic reticulum (ER) of the preoperative hepatocytes were normal. Within a day after HIRI, however, significant ultrastructural changes were observed, such as nuclear membrane shrinkage, chromatin condensation at the edges, mitochondrial swelling, and severe ER expansion. These changes were less evident 3 days postoperation and largely subsided by day 7 with only a slight expansion of the ER. Transplantation of either ADSCs or the ADSC-secretome significantly alleviated 


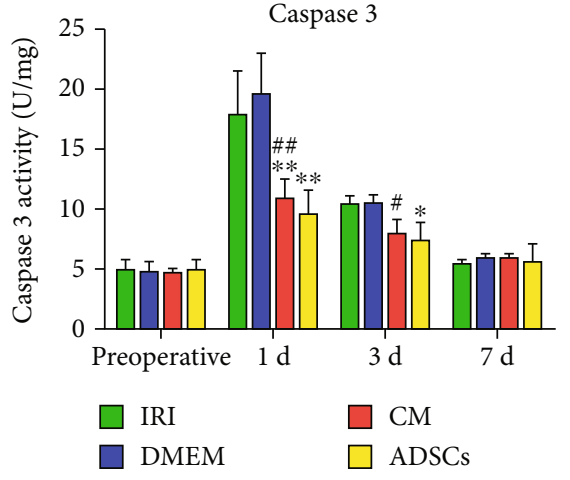

(a)

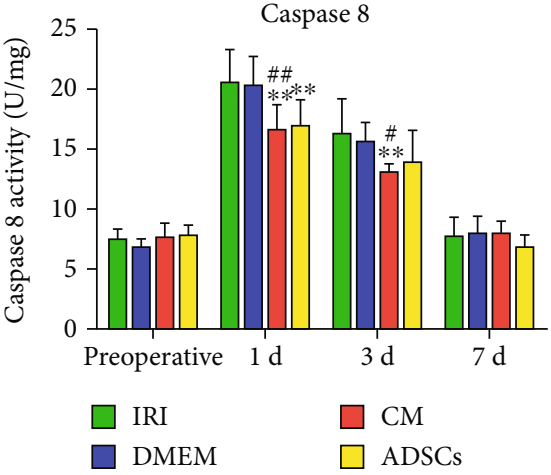

(b)

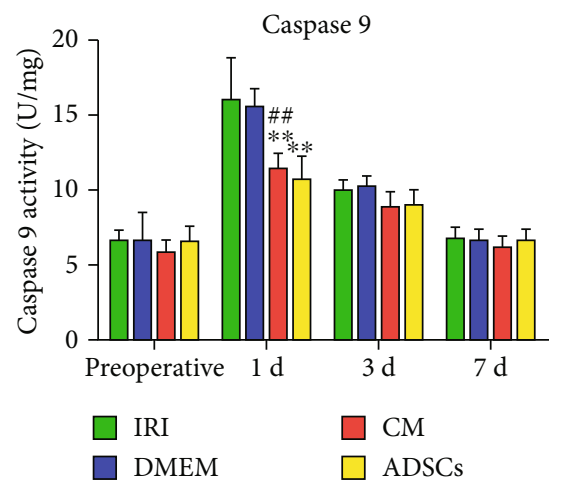

(c)

Figure 3: Caspase activity levels: (a) caspase 3, (b) caspase 8, and (c) caspase $9 .{ }^{*} P<0.05$ and ${ }^{* *} P<0.01$ compared to IRI group; ${ }^{*} P<0.05$ and ${ }^{\# \#} P<0.01$ compared to DMEM group.

mitochondrial swelling and ER expansion postoperation, whereas DMEM had no effect. The results indicate that ADSCs/ADSC-secretome can improve the ultrastructural changes in hepatocytes after ischemia-reperfusion and partial hepatectomy.

\subsection{ADSCs/ADSC-Secretome Decreased Postischemic} Hepatocyte Apoptosis. As shown in Figure 2(a), numerous TUNEL-positive apoptotic cells were present in the hepatic tissues 1 and 3 days after surgery. The apoptosis rates at both time points were significantly higher in the untreated and DMEM control animals compared to those transplanted with ADSCs and the ADSC-secretome (Figure 2(b); $P<0.01$ ). Thus, the ADSCs and their secretome can alleviate IRIinduced apoptosis in the hepatocytes.

3.3. ADSCs/ADSC-Secretome Decreased Caspase Activity in Hepatocytes. To further elucidate the mechanistic basis of the antiapoptotic effects of ADSCs/ADSC-secretome, we analyzed the activity of multiple caspases in the liver tissues after ischemia-reperfusion and partial hepatectomy. As shown in Figure 3, the activity of caspase 3, caspase 8, and caspase 9 peaked 1 day after surgery but was significantly reduced in the ADSCs/CM-treated groups $(P<0.01)$. In addition, caspase 8 activity remained significantly higher on day 3 postoperation in the IRI and DMEM control groups compared to the CM-treated group $(P<0.01, P<0.05)$. Caspase 3 activity levels dropped by day 3 even in the untreated groups, although the reduction was more significant in the CM-treated versus DMEM groups $(P<0.05)$. In contrast, caspase 9 activity was similar across all groups 3 days after operation. Caspase activity levels were restored to normal in the untreated animals at day 7 postoperation.

3.4. ADSCs/ADSC-Secretome Altered the Expression of Apoptosis-Related Factors. The antiapoptotic effects of the ADSCs/ADSC-secretome were further confirmed by analyzing the expression levels of apoptosis-related proteins including Bax, Bcl-2, P53, Fas, and Fasl. As shown in Figure 4(a), Bax mRNA levels increased significantly after surgery and were downregulated by both ADSCs and the ADSCsecretome on day $1(P<0.01)$ and day $3(P<0.05)$ postoperation. On the other hand, the antiapoptotic Bcl-2 was downregulated after surgery and increased in the animals treated with ADSCs/ADSC-secretome on days 1 and 3 postoperation $(P<0.01$ compared to the DMEM group; Figure 4(b)). Consistent with this, the ADSCs and ADSCsecretome significantly decreased the $\mathrm{Bax} / \mathrm{Bcl}-2$ ratio at both time points $(P<0.01$, Figure $4(\mathrm{c}))$. The upstream regulator P53 was also downregulated by the ADSC-secretome and ADSCs on days $1(P<0.01$ for both) and $3(P<0.05$ and $P$ $<0.01)$ compared to the untreated IRI and DMEM groups (Figure 4(d)). The Fas and Fasl transcripts also showed similar trends $(P<0.01$ for all; Figures $4(\mathrm{e})$ and $4(\mathrm{f}))$. Taken together, the ADSCs and ADSC-secretome upregulated the antiapoptotic genes and suppressed the proapoptotic genes 


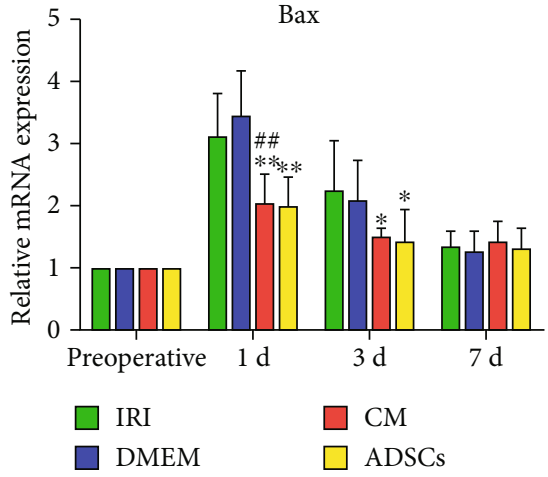

(a)

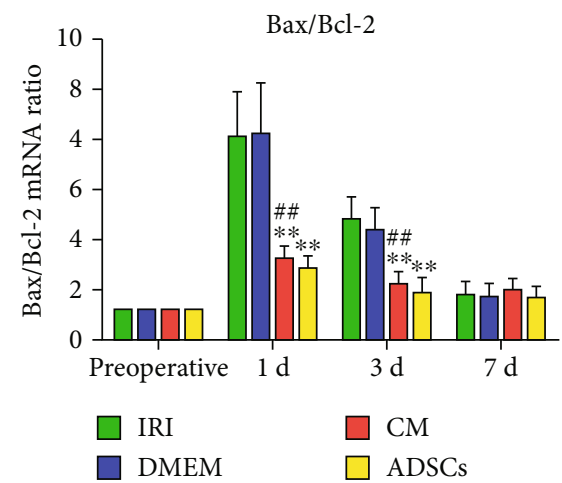

(c)

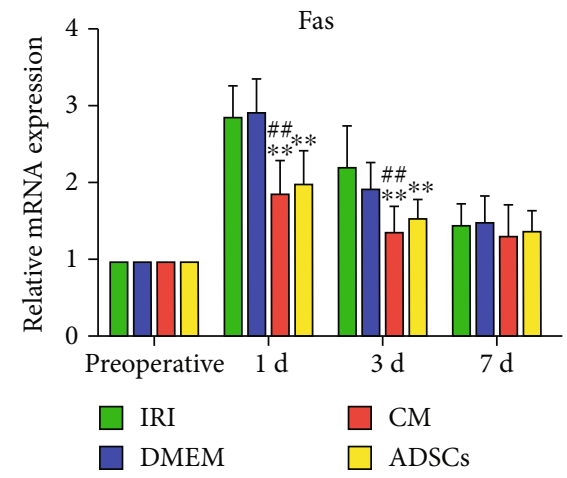

(e)

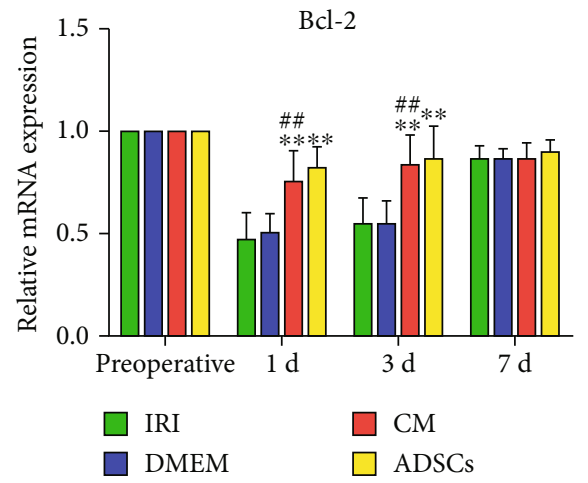

(b)

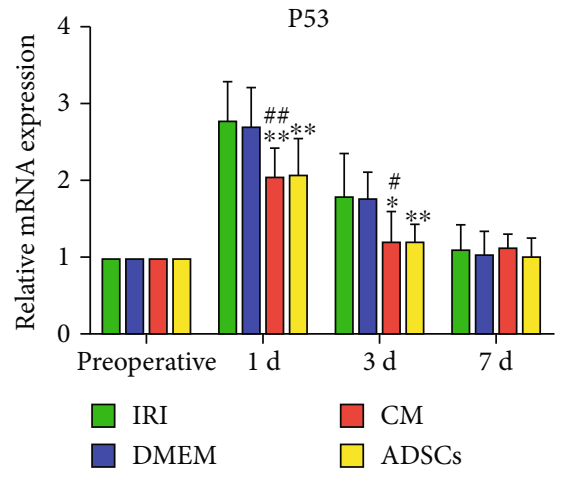

(d)

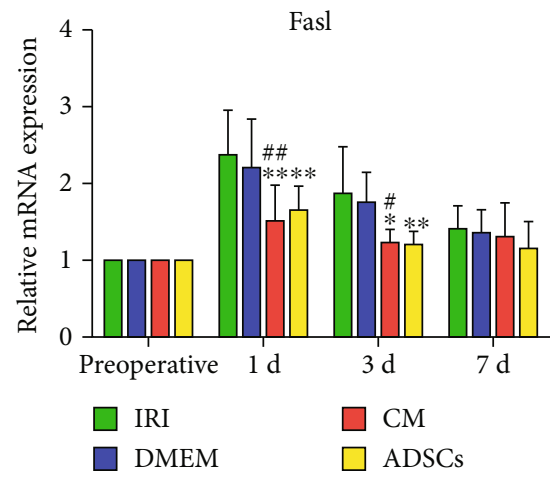

(f)

Figure 4: Expression levels of apoptosis-related genes in liver tissue. ${ }^{*} P<0.05$ and ${ }^{* *} P<0.01$ compared to IRI group; ${ }^{*} P<0.05$ and ${ }^{\# \#} P<0.01$ compared to DMEM group.

in hepatocytes. Consistent with the above, cytoplasmic expression of Fas and Fasl proteins increased in the liver parenchyma of all groups after surgery (Figures $5(\mathrm{a})$ and 5(b)). However, both ADSCs and the ADSC-secretome significantly reduced the in situ expression of both on days 1 and 3 after surgery $(P<0.01$ and $P<0.05$, respectively, as indicated in Figures 5(c) and 5(d)).

Likewise, the ADSCs and the ADSC-secretome also significantly reduced $\mathrm{P} 53$ and Bax protein expression on days $1(P<0.01$ and $P<0.05$, respectively) and $3(P<0.05$ and $P<0.01$, respectively) after surgery (Figures $6(\mathrm{a})-6(\mathrm{c})$ ) and upregulated $\mathrm{Bcl}-2$ at both time points $(P<0.01$; Figures 6(a) and 6(d)). Consistent with this, the Bax/Bcl-2 ratio was significantly lower in the ADSC/CM-treated ani- mals compared to the untreated IRI and DMEM control on days 1 and $3(P<0.01)$ postoperation (Figure $6(\mathrm{e}))$.

\section{Discussion}

Laparoscopic hepatectomy has been successfully used to establish liver injury in large animal models [32,33]. Multiple studies show that the stem cell-derived secretome plays an active role in alleviating the symptoms of ischemiareperfusion [34-36]. However, it is unclear whether the ADSC-secretome in particular exerts an active therapeutic effect on HIRI. Therefore, the aim of our study was to evaluate the effect of ADSCs and its secretome on hepatocyte apoptosis after HIRI combined with partial hepatectomy. 

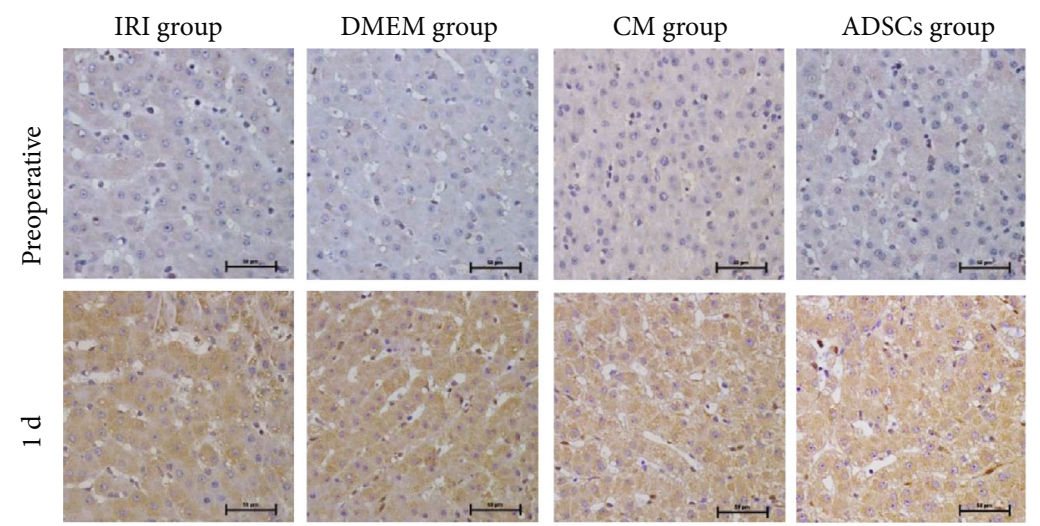

ro
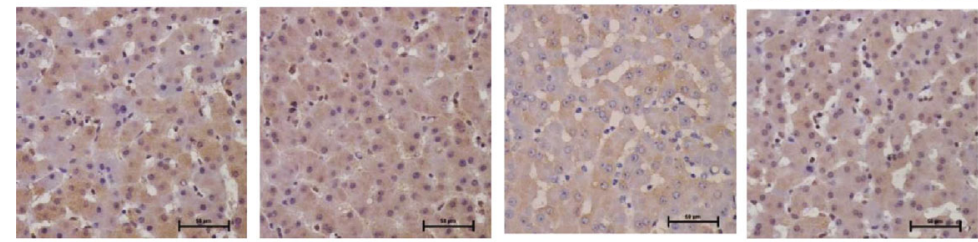

$\nabla$
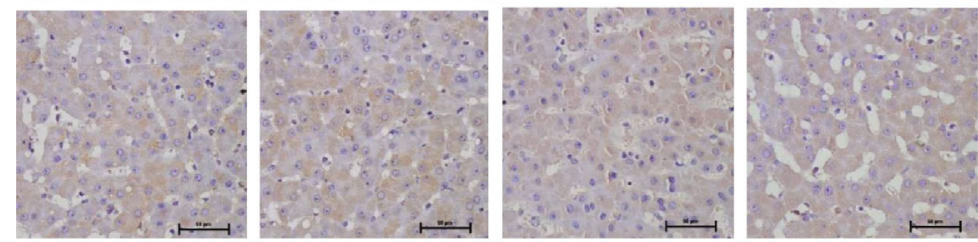

(a)
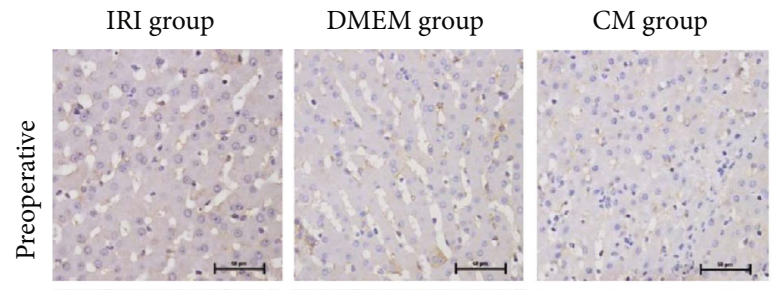

ADSCs group
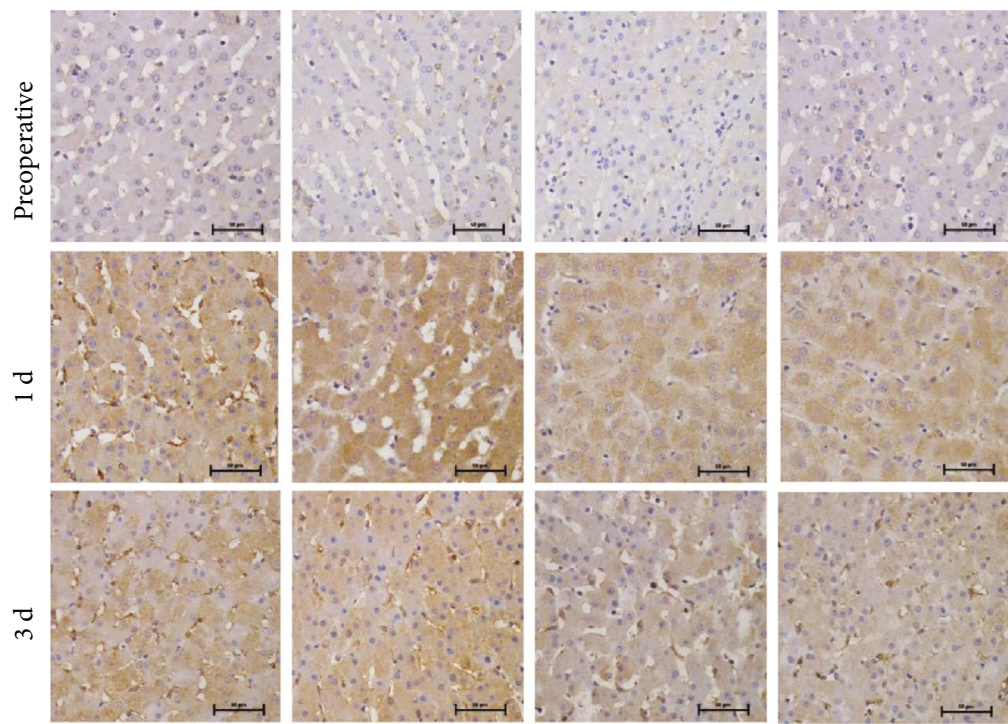

문
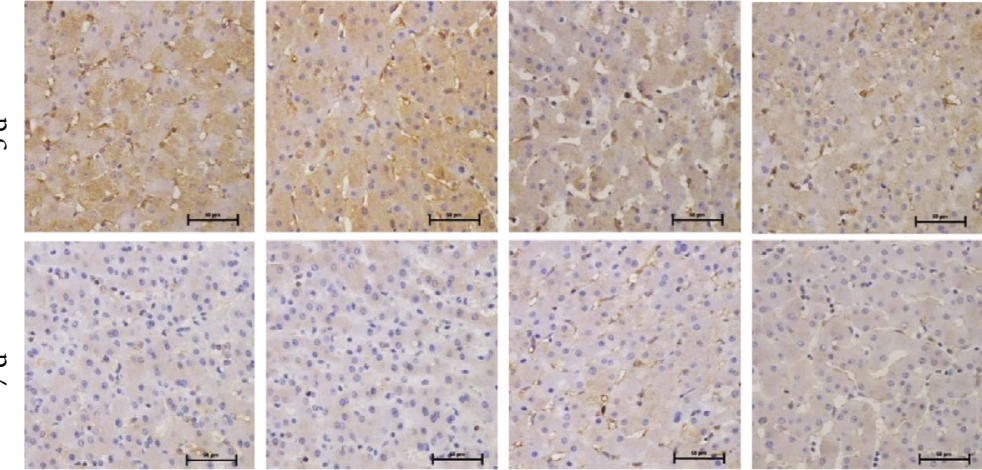

(b)

Figure 5: Continued. 


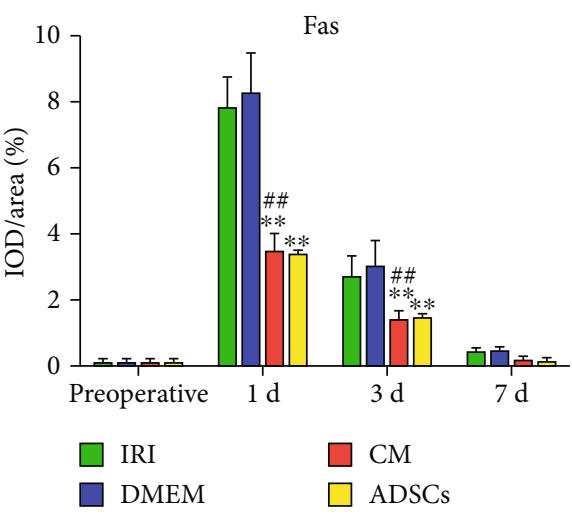

(c)

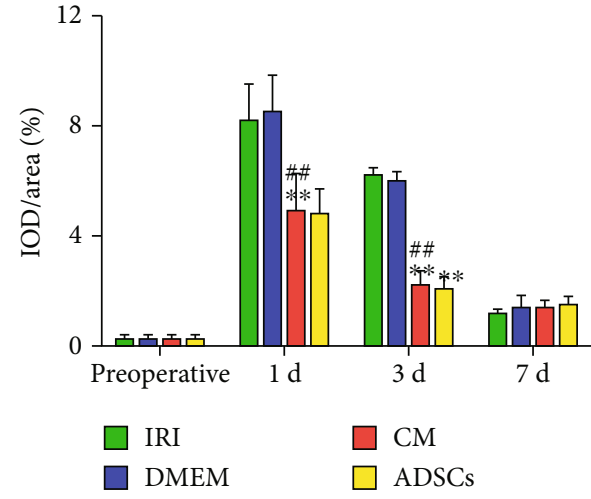

(d)

FIGURE 5: In situ expression levels of Fas and Fasl protein in liver tissues. (a, b) Representative images of immunohistochemically stained Fas and Fasl protein in the indicated groups (magnification 400x). (c, d) Relative expression levels of Fas and Fasl. ${ }^{*} P<0.05$ and ${ }^{* *} P<0.01$ compared to IRI group; ${ }^{\#} P<0.05$ and ${ }^{\# \#} P<0.01$ compared to DMEM group.

ADSCs are adult stem cells with immune regulation, secretion of growth factors, promotion of blood vessel formation, and tissue regeneration. Compared with the secretome from other stem cells, the ADSC-secretome has obvious advantages, including no bioethical restrictions of embryonic stem cells, large-scale production, easy storage and transportation, and fast therapeutic effect. So, it is economical and practical in clinical practice and brings hope to the application of cell-free therapy. The miniature pig is a kind of experimental animal with abundant adipose tissue. In addition, miniature pigs are suitable experimental animals for studying pathological changes in organs due to the anatomical and physiological similarities with humans. ADSCs from miniature pigs secrete proteins such as ANG-1, ANG-2, VEGF, and b-FGF [37]. Previous studies have demonstrated that ANG-1 can promote the expression of the Bcl-2 protein [38]. b-FGF can participate in the process of cellular mitosis and induce cell proliferation and differentiation which prevent cell apoptosis [39]. Therefore, the secreted protein may have a role in the antiapoptotic effect of the ADSCsecretome. TUNEL staining is a shared method to detect cell apoptosis. After HIRI, TUNEL staining showed an increase in the number of apoptotic liver cells [40]. Yi-Xing et al. found that MSC-CM has a direct inhibitory effect on sinusoidal endothelial cell apoptosis by TUNEL staining [41]. And the CM of human umbilical cord mesenchymal stem cells can also reduce the percentage of TUNEL-positive cells to play a protective effect on the cells [42]. Similarly, our results show that the ADSC-secretome from miniature pigs reduced the numbers of apoptosis cells by TUNEL staining after HIRI combined with partial hepatectomy.

HIRI is a complex pathophysiological process that involves ischemia, hypoxia, early reperfusion, and reperfusion injury. Liver tissue reperfusion generates a large amount of ROS, and the resulting oxidative stress accelerates tissue inflammation and cell death [43]. In addition, the $\mathrm{Ca}^{2+}$ overload during ischemia-reperfusion alters mitochondrial membrane permeability, which lowers ATP production and oxygen consumption, thereby affecting the survival of liver cells. Apoptosis was first described by Kerr [44] in hepatocytes. We detected a significant increase in apoptotic cells in the liver tissue after ischemia-reperfusion, which correlated to ultrastructural changes such as chromatin disintegration, mitochondrial swelling, and endoplasmic reticulum expansion. Although antioxidants improve the symptoms of HIRI, they are not feasible for clinical application [45]. MSC-CM protects cells from apoptosis $[42,46]$ and can improve mitochondrial function and reduce hepatocyte apoptosis in nonalcoholic fatty liver disease [47]. In addition, previous studies have demonstrated antioxidative and antiinflammatory effects of ADSCs $[11,37]$. Consistent with this, both ADSCs and the ADSC-secretome alleviated apoptosis following HIRI, as indicated by improved organelle structure, lower levels of caspases, and downregulation of proapoptotic genes and proteins.

Hepatocyte apoptosis is involved in maintaining the normal physiological functions of the liver and plays an important role in acute or chronic diseases of the liver, such as I/R injury, viral hepatitis, alcoholic and nonalcoholic liver diseases, and cholestatic diseases [48]. Therefore, understanding the mechanism of hepatocyte apoptosis is of great significance for the treatment of liver diseases. Apoptosis is mediated via the endogenous mitochondrial pathway, exogenous death receptor pathway, and endoplasmic reticulum pathway and regulated by the caspase family, Bcl-2, and P53 among others. The P53 protein forms a complex that transports the Bax protein to the nucleus, which promotes Bax expression and inhibits Bcl-2 to mediate early apoptosis [49]. Furthermore, various death signals depolarize the mitochondrial membrane by opening the transition pore, which releases cytochrome $\mathrm{C}$ into the cytoplasm. Cytochrome $\mathrm{C}$ forms multimers with Apaf1 and ATP/d ATP, which activate the caspase 9 precursor by promoting self-cleavage. Cleaved caspase 9 triggers the downstream caspase 3 and caspase 7 cascade, eventually culminating in apoptosis. A previous study showed that the $\mathrm{CM}$ of bone marrow mesenchymal stem cells (BMSCs) alleviated neuronal apoptosis by downregulating Bax and the cleaved caspase 3/caspase ratio and 


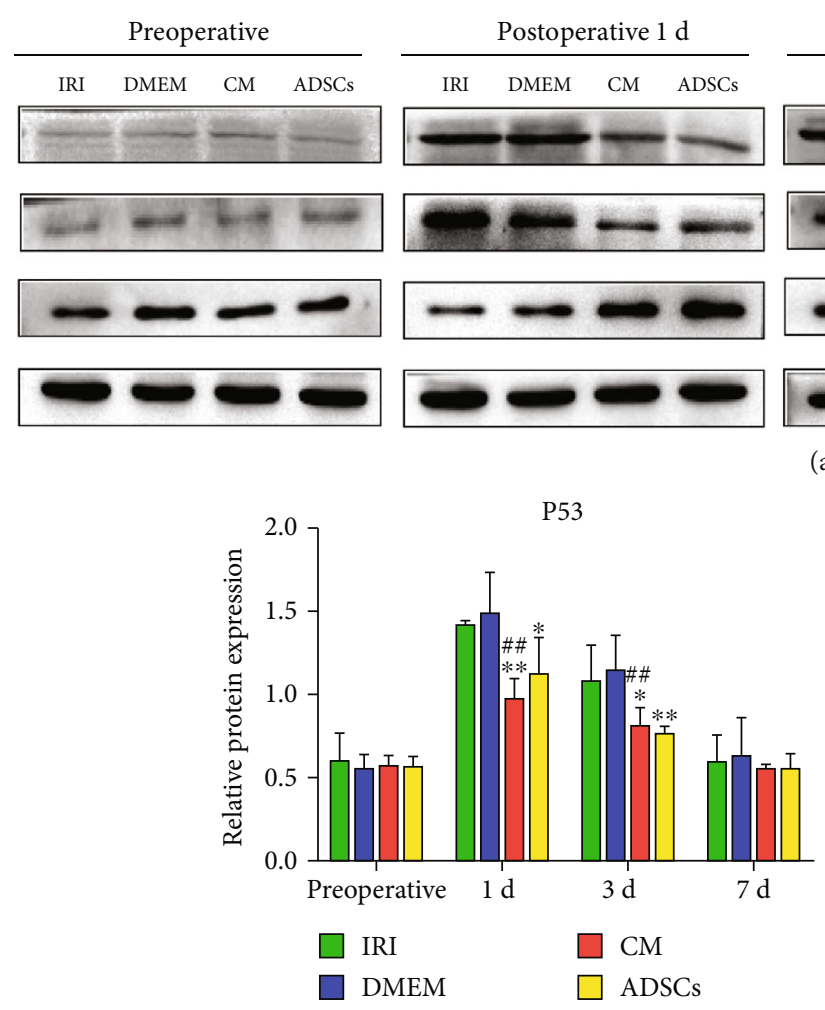

(b)

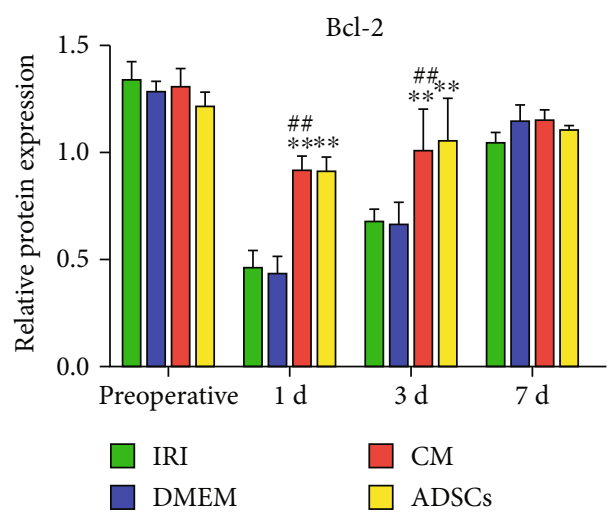

(d)
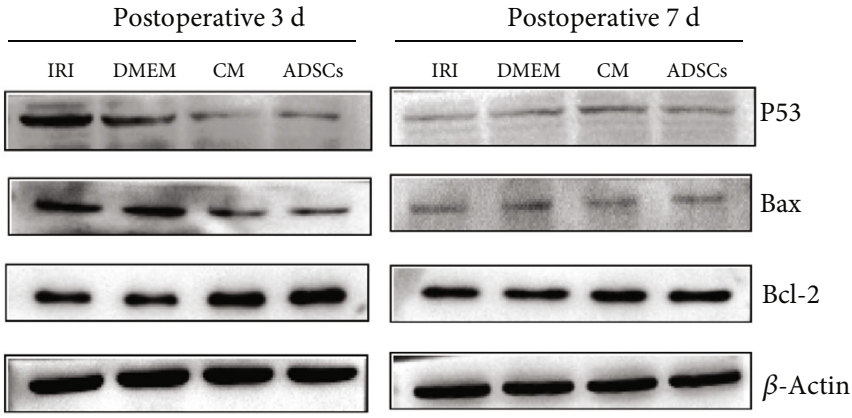

(a)

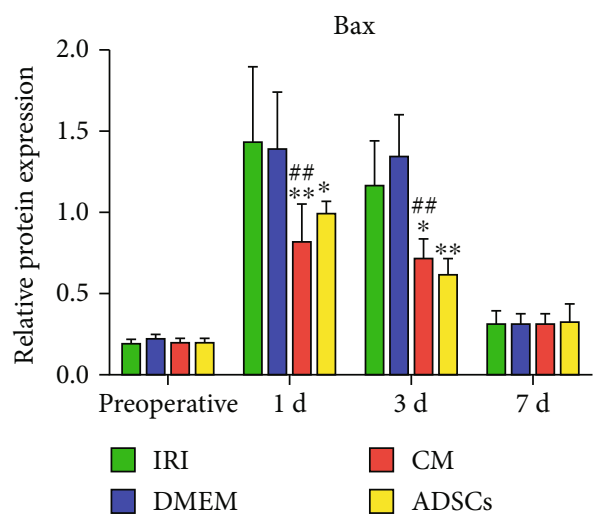

(c)

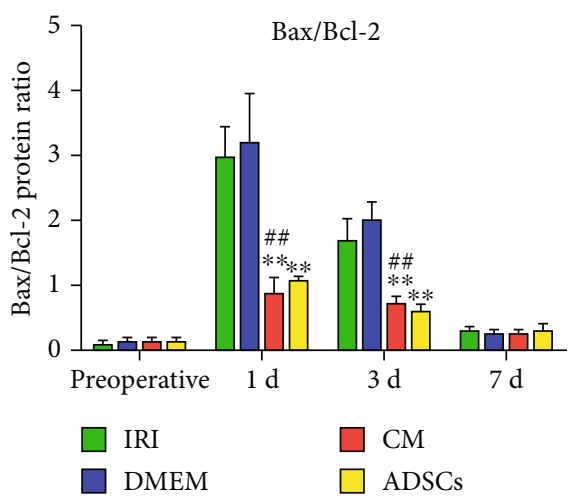

(e)

FiguRE 6: Expression levels of apoptosis-related proteins in liver tissue: (a) representative immunoblot showing P53, Bax, Bcl-2, and $\beta$-actin levels in the indicated groups. (b-e) Quantification of P53, Bax, Bcl-2, and Bax/Bcl-2 ratio. ${ }^{*} P<0.05$ and ${ }^{* *} P<0.01$ compared to IRI group; ${ }^{\#} P<0.05$ and ${ }^{\# \#} P<0.01$ compared to DMEM group.

increasing Bcl-2 levels [50]. The Bcl-2 protein family is currently the most valuable protein family that regulates apoptosis. Antiapoptotic proteins $\mathrm{Bcl}-2, \mathrm{Bcl}-\mathrm{x}$, and $\mathrm{Bcl}-\mathrm{w}$ block the apoptotic cascade by inhibiting cytochrome $\mathrm{C}$ release [51]. Another study demonstrated that ADSC-CM can significantly reduce the expression of proapoptotic proteins such as Bax during ischemia-reperfusion-induced cardiac injury [52]. P53 directly activates Bax to permeabilize the mitochondrial membrane and initiate the apoptotic program [51]. In our study, ADSCs and the ADSC-secretome downregulated P53 and Bax levels in the injured hepatocytes, reduced caspase 3 and 9 activity, and upregulated $\mathrm{Bcl}-2$ following liver ischemia-reperfusion.
The exogenous death receptor apoptosis pathway includes the tumor necrosis factor receptor (TNFR) signaling pathway, TNF-related apoptosis-inducing ligand (TRAIL) signaling pathway, and Fas ligand (Fas/Fasl) signaling pathway [29]. Studies show that all three receptors can activate caspase 8 after binding to their corresponding ligands, resulting in caspase 8 and caspase 3 cleavage causing cell apoptosis [53]. Among them, the Fas/Fasl pathway is the most detailed study of the death receptor family. Following Fas-Fasl binding, Fas undergoes trimerization, and its death domain activates caspase 8, which subsequently triggers the apoptotic cascade. Previous studies have shown that Fas and Fasl expression levels, as well as caspase 8 activity, increased rapidly after 
liver ischemia-reperfusion and partial hepatectomy [20]. We found that ADSCs and the ADSC-secretome significantly reduced the expression of the above factors. Consistent with our findings, Kappy found that human ADSC-derived CM protected neuroblastoma cells from apoptosis by significantly reducing Fas expression levels [54]. In addition, MSC-CM effectively reduced radiation-induced apoptosis in hepatic sinusoidal endothelial cells [41], and BMSC-CM alleviated hepatocyte apoptosis in the carbon tetrachloride-induced acute liver injury mouse model [55]. Thus, the secretome of stem cells can target both endogenous and exogenous apoptotic pathways.

At present, the route of administration of stem cells, secretome, and exosomes include systemic administration and local administration [56]. Intravenous injection is a better method of systemic administration for experimental animals, but the effective ingredients that home to the liver via the peripheral venous blood are limited, so it takes a long time to exert a therapeutic effect. The procedure of portal vein injection in large animals is complicated. In addition, there is a risk of vascular embolism, and most of the stem cells may be cleared by the liver in the early stage after portal vein injection [57]. Liver parenchymal injection for ADSC and ADSC-secretome transplantation was used in this study. However, this method of administration is also a kind of damage to the liver. Therefore, it is necessary to develop a new route of administration which should be simple to operate, widely used, safe, and effective, such as noninvasive nasal inhalation [58] or dressing in combination with a hydrogel [9]. Nevertheless, the ADSC-secretome injected into the liver parenchyma still exerts an antiapoptotic effect after HIRI combined with partial hepatectomy.

\section{Conclusion}

The ADSCs and ADSC-secretome can mitigate liver injury after HIRI combined with partial hepatectomy by blocking the endogenous and exogenous apoptotic pathways. Our findings indicate that the ADSC-secretome can inhibit hepatocyte apoptosis. The paracrine therapeutic effects of ADSCs are mediated by their secretome. Therefore, the ADSCsecretome can overcome the limitations of cell-based therapies which is a viable alternative for stem cell-based tissue repair and regeneration.

\section{Data Availability}

The datasets used and/or analyzed during this study are available from the corresponding author upon reasonable request.

\section{Conflicts of Interest}

The authors declare no conflict of interest.

\section{Acknowledgments}

The authors thank Hongbin Wang who designed this study and revised the manuscript. The authors also thank the Heilongjiang Key Laboratory for Laboratory Animals and
Comparative Medicine. This research was funded by the National Natural Science Foundation of China, grant numbers 31972757 and 31772807 .

\section{References}

[1] A. Panisello-Roselló and J. Roselló-Catafau, "Molecular Mechanisms and Pathophysiology of Ischemia-Reperfusion Injury," International Journal of Molecular Sciences, vol. 19, no. 12, p. 4093, 2018.

[2] M. Cour, J. Loufouat, M. Paillard et al., "Inhibition of mitochondrial permeability transition to prevent the post-cardiac arrest syndrome: a pre-clinical study," European Heart Journal, vol. 32, no. 2, pp. 226-235, 2011.

[3] C. Li, K. T. P. Ng, Y. Shao et al., "The inhibition of aldose reductase attenuates hepatic ischemia-reperfusion injury through reducing inflammatory response," Annals of Surgery, vol. 260, no. 2, pp. 317-328, 2014.

[4] Y. ZHAI, R. W. BUSUTTIL, and J. W. KUPIEC-WEGLINSKI, "Liver ischemia and reperfusion injury: new insights into mechanisms of innate-adaptive immune-mediated tissue inflammation," American Journal of Transplantation, vol. 11, no. 8, pp. 1563-1569, 2011.

[5] N. C. TEOH and G. C. FARRELL, "Hepatic ischemia reperfusion injury: pathogenic mechanisms and basis for hepatoprotection," Journal of Gastroenterology and Hepatology, vol. 18, no. 8, pp. 891-902, 2003.

[6] H. Hirao, K. J. Dery, S. Kageyama, K. Nakamura, and J. W. Kupiec-Weglinski, "Heme Oxygenase-1 in liver transplant ischemia-reperfusion injury: From bench- to-bedside," Free Radical Biology and Medicine, vol. 157, pp. 75-82, 2020.

[7] L. Trovato, F. Naro, F. D’Aiuto, and F. Moreno, "Promoting tissue repair by micrograft stem cells delivery," Stem cells international, vol. 2020, Article ID 2195318, 2 pages, 2020.

[8] Z. Hu, Y. Xia, S. G. Hong, E. Thomas, and D. Li, "Stem Cells and Liver Diseases," Stem Cells International, vol. 2019, Article ID 9271746, 2 pages, 2019.

[9] F. Poustchi, H. Amani, Z. Ahmadian et al., "Combination therapy of killing diseases by injectable hydrogels: from concept to medical applications," Advanced Healthcare Materials, vol. 10, no. 3, 2021.

[10] C. Michaeloudes, X. Li, J. C. W. Mak, and P. K. Bhavsar, "Study of mesenchymal stem cell-mediated mitochondrial transfer in in vitro models of oxidant-mediated airway epithelial and smooth muscle cell injury," Methods in molecular biology, vol. 2269, pp. 93-105, 2021.

[11] Y. Ge, Q. Zhang, Z. Jiao, H. Li, G. Bai, and H. Wang, “Adiposederived stem cells reduce liver oxidative stress and autophagy induced by ischemia-reperfusion and hepatectomy injury in swine," Life sciences, vol. 214, pp. 62-69, 2018.

[12] J. Oliva, "Therapeutic properties of mesenchymal stem cell on organ ischemia-reperfusion injury," International Journal of Molecular Sciences, vol. 20, no. 21, p. 5511, 2019.

[13] Z. Jiao, Y. Ma, X. Liu et al., "Adipose-derived stem cell transplantation attenuates inflammation and promotes liver regeneration after ischemia-reperfusion and hemihepatectomy in swine," Stem Cells International, vol. 2019, Article ID 2489584, 13 pages, 2019.

[14] S. Gaus, H. Li, S. Li et al., "Shared genetic and epigenetic mechanisms between the osteogenic differentiation of dental pulp 
stem cells and bone marrow stem cells," BioMed research international, vol. 2021, Article ID 6697810, 25 pages, 2021.

[15] A. SHOJAEIAN, A. MEHRI-GHAHFARROKHI, and M. BANITALEBI-DEHKORDI, "Migration gene expression of human umbilical cord mesenchymal stem cells: a comparison between monophosphoryl lipid A and supernatant of Lactobacillus acidophilus," International Journal of Molecular and Cellular Medicine, vol. 8, no. 2, p. 2, 2019.

[16] Y. Lee, S. H. Shin, K. A. Cho et al., "Administration of tonsil-derived mesenchymal stem cells improves glucose tolerance in high fat diet-induced diabetic mice via insulin-like growth factor-binding protein 5-mediated endoplasmic reticulum stress modulation," Cell, vol. 8 , no. 4, p. 368, 2019.

[17] L. L. Zhou, W. Liu, Y. M. Wu, W. L. Sun, C. E. Dörfer, and K. M. Fawzy el-Sayed, "Oral mesenchymal stem/progenitor cells: the immunomodulatory masters," Stem cells international, vol. 2020, Article ID 1327405, 16 pages, 2020.

[18] A. T. Wang, Q. F. Zhang, N. X. Wang et al., "Cocktail of hyaluronic acid and human amniotic mesenchymal cells effectively repairs cartilage injuries in sodium iodoacetateinduced osteoarthritis rats," Frontiers in Bioengineering and Biotechnology, vol. 8, 2020.

[19] N. Dubey, V. Mishra, R. Dubey, Y. H. Deng, F. C. Tsai, and W. P. Deng, "Revisiting the advances in isolation, characterization and secretome of adipose-derived stromal/stem cells," International journal of molecular sciences, vol. 19, no. 8, p. 2200, 2018.

[20] Y. Ge, Q. Zhang, H. Li, G. Bai, Z. Jiao, and H. Wang, "Adiposederived stem cells alleviate liver apoptosis induced by ischemia- reperfusion and laparoscopic hepatectomy in swine," Scientific Reports, vol. 8, no. 1, 2018.

[21] V. Volarevic, B. S. Markovic, M. Gazdic et al., "Ethical and safety issues of stem cell-based therapy," International Journal of Medical Sciences, vol. 15, no. 1, pp. 36-45, 2018.

[22] A. Karnoub, A. B. Dash, A. P. Vo et al., "Mesenchymal stem cells within tumour stroma promote breast cancer metastasis," Nature, vol. 449, no. 7162, pp. 557-563, 2007.

[23] L. Barrachina, A. Romero, P. Zaragoza, C. Rodellar, and F. J. Vázquez, "Practical considerations for clinical use of mesenchymal stem cells: from the laboratory to the horse," The Veterinary Journal, vol. 238, pp. 49-57, 2018.

[24] B. Lukomska, L. Stanaszek, E. Zuba-Surma, P. Legosz, S. Sarzynska, and K. Drela, "Challenges and controversies in human mesenchymal stem cell therapy," Stem cells international, vol. 2019, 10 pages, 2019.

[25] A. Hassanzadeh, H. S. Rahman, A. Markov et al., "Mesenchymal stem/stromal cell-derived exosomes in regenerative medicine and cancer; overview of development, challenges, and opportunities," Stem Cell Research \& Therapy, vol. 12, no. 1, p. 297, 2021.

[26] A. G. Zhao, K. Shah, B. Cromer, and H. Sumer, "Mesenchymal stem cell-derived extracellular vesicles and their therapeutic potential," Stem cells international, vol. 2020, Article ID 8825771, 10 pages, 2020.

[27] A. RATUSHNYY, M. EZDAKOVA, and L. BURAVKOVA, "Secretome of senescent adipose-derived mesenchymal stem cells negatively regulates angiogenesis," International Journal of Molecular Sciences, vol. 21, no. 5, p. 1802, 2020.

[28] A. T. Brini, G. Amodeo, L. M. Ferreira et al., "Therapeutic effect of human adipose-derived stem cells and their secretome in experimental diabetic pain," Scientific Reports, vol. 7, no. 1, p. $9904,2017$.

[29] X. Ni, X. Shan, L. Xu et al., "Adipose-derived stem cells combined with platelet-rich plasma enhance wound healing in a rat model of full-thickness skin defects," Stem Cell Research \& Therapy, vol. 12, no. 1, p. 226, 2021.

[30] M. Ishida, K. Tatsumi, K. Okumoto, and H. Kaji, “Adipose tissue-derived stem cell sheet improves glucose metabolism in obese mice," Stem Cells and Development, vol. 29, no. 8, pp. 488-497, 2020.

[31] Z. Jiao, X. Liu, Y. Ma et al., "Adipose-derived stem cells protect ischemia-reperfusion and partial hepatectomy by attenuating endoplasmic reticulum stress," Frontiers in Cell and Developmental Biology, vol. 8, no. 177, 2020.

[32] H. Li, G. Bai, Y. Ge et al., "Hydrogen-rich saline protects against small-scale liver ischemia-reperfusion injury by inhibiting endoplasmic reticulum stress," Life sciences, vol. 194, pp. 7-14, 2018.

[33] Q. Zhang, C. Piao, J. Xu et al., "Comparative study on protective effect of hydrogen rich saline and adipose- derived stem cells on hepatic ischemia-reperfusion and hepatectomy injury in swine," Biomedicine \& Pharmacotherapy, vol. 120, p. 109453, 2019.

[34] S. C. Lee, K. H. Kim, O. H. Kim et al., "Determination of optimized oxygen partial pressure to maximize the liver regenerative potential of the secretome obtained from adipose-derived stem cells," Stem cell research \& therapy, vol. 8, no. 1, p. 181, 2017.

[35] X. Li, T. Ma, J. Sun et al., "Harnessing the secretome of adipose-derived stem cells in the treatment of ischemic heart diseases," Stem Cell Research \& Therapy, vol. 10, no. 1, p. 196, 2019.

[36] F. Chen, B. Liu, Y. Y. Ming et al., "Conditioned medium of bone marrow mesenchymal stem cells via intravenous injection to treat cerebral ischemia-reperfusion injury," Chinese Journal of Tissue Engineering Research, vol. 28, pp. 45444548, 2015.

[37] Z. Jiao, Y. Ma, Q. Zhang et al., "The adipose-derived mesenchymal stem cell secretome promotes hepatic regeneration in miniature pigs after liver ischaemia-reperfusion combined with partial resection," Stem Cell Research \& Therapy, vol. 12, no. 1, 2021.

[38] S. LIAN-GUO, Z. GUO-PING, and J. Huiming, "Inhibition of microvascular endothelial cell apoptosis by angiopoietin-1 and the involvement of cytochrome C," Chinese Medical Journal, vol. 119, no. 9, 2006.

[39] K. Ladeira, F. Macedo, A. Longatto-Filho, and S. F. Martins, "Angiogenic factors: role in esophageal cancer, a brief review," Esophagus, vol. 15, no. 2, pp. 53-58, 2018.

[40] J. Lin, H. F. Huang, S. K. Yang et al., "The effect of ginsenoside Rg1 in hepatic ischemia reperfusion (I/R) injury ameliorates ischemia-reperfusion-induced liver injury by inhibiting apoptosis," Biomedicine \& Pharmacotherapy, vol. 129, p. 110398, 2020.

[41] Y.-X. Chen, Z.-C. Zeng, J. Sun, H.-Y. Zeng, Y. Huang, and Z.Y. Zhang, "Mesenchymal stem cell-conditioned medium prevents radiation-induced liver injury by inhibiting inflammation and protecting sinusoidal endothelial cells," Journal of Radiation Research, vol. 56, no. 4, pp. 700-708, 2015.

[42] L. Hong, L. Yan, Z. Xin et al., "Protective effects of human umbilical cord mesenchymal stem cell-derived conditioned 
medium on ovarian damage," Journal of Molecular Cell Biology, vol. 12, no. 5, pp. 372-385, 2020.

[43] P. Bystrom, N. Foley, L. Toledo-Pereyra, and K. Quesnelle, "Ischemic preconditioning modulates ROS to confer protection in liver ischemia and reperfusion," EXCLI journal, vol. 16, p. 483, 2017.

[44] J. Kerr, A. H. Wyllie, and A. R. Currie, “Apoptosis: a basic biological phenomenon with wideranging implications in tissue kinetics," British Journal of Cancer, vol. 26, no. 4, pp. 239257, 1972.

[45] H. M. Cochemé and M. P. Murphy, "Can antioxidants be effective therapeutics?," Current Opinion in Investigational Drugs, vol. 11, no. 4, pp. 426-431, 2010.

[46] S. N. Maleki, N. Aboutaleb, D. Nazarinia, S. A. Beik, A. Qolamian, and M. Nobakht, "Conditioned medium obtained from human amniotic membrane-derived mesenchymal stem cell attenuates heart failure injury in rats," Iranian Journal of Basic Medical Sciences, vol. 22, no. 11, pp. 1253-1258, 2019.

[47] M. Yang, Y. Cui, J. Song et al., "Mesenchymal stem cellconditioned medium improved mitochondrial function and alleviated inflammation and apoptosis in non-alcoholic fatty liver disease by regulating SIRT1," Biochemical and biophysical research communications, vol. 546, pp. 74-82, 2021.

[48] L. Cao, X. B. Quan, W. J. Zeng, X. O. Yang, and M. J. Wang, "Mechanism of hepatocyte apoptosis," Journal of Cell Death, vol. 9, p. JCD.S39824, 2016.

[49] J. Chipuk, T. Kuwana, L. Bouchier-Hayes et al., "Direct activation of Bax by 553 mediates mitochondrial membrane permeabilization and apoptosis," Science, vol. 303, no. 5660, pp. 1010-1014, 2004.

[50] C. Wen, C. Huang, M. Yang et al., "The secretion from bone marrow mesenchymal stem cells pretreated with berberine rescues neurons with oxidative damage through activation of the Keap1-Nrf2-HO-1 signaling pathway," Neurotoxicity Research, vol. 38, no. 1, pp. 59-73, 2020.

[51] M. Lam, M. B. Bhat, G. Nuñez, J. Ma, and C. W. Distelhorst, "Regulation of Bcl-xl channel activity by calcium," Journal of Biological Chemistry, vol. 273, no. 28, pp. 17307-17310, 1998.

[52] T. L. Lee, T. C. Lai, S. R. Lin et al., "Conditioned medium from adipose-derived stem cells attenuates ischemia/reperfusioninduced cardiac injury through the microRNA221/222/PUMA/ETS-1 pathway," Theranostics, vol. 11, no. 7, pp. 3131-3149, 2021.

[53] L. J. Liu, J. X. Peng, H. Z. Hong, W. Ye, and Y. Y. Qiao, "Mitochondrial changes and role in apoptosis," Chinese Journal of Cell Biology, vol. 27, no. 2, pp. 117-120, 2005.

[54] N. Kappy, S. Chang, W. M. Harris et al., "Human adiposederived stem cell treatment modulates cellular protection in both in vitro and in vivo traumatic brain injury models," Journal of Trauma and Acute Care Surgery, vol. 84, no. 5, pp. 745751, 2018.

[55] A. Xagorari, E. Siotou, M. Yiangou et al., "Protective effect of mesenchymal stem cell-conditioned medium on hepatic cell apoptosis after acute liver injury," International Journal of Clinical and Experimental Pathology, vol. 6, no. 5, pp. 831840, 2013.

[56] F. Shekari, A. Nazari, S. Assar Kashani, E. Hajizadeh-Saffar, R. Lim, and H. Baharvand, "Pre-clinical investigation of mesenchymal stromal cell-derived extracellular vesicles: a systematic review," Cytotherapy, vol. 23, no. 4, pp. 277-284, 2021.
[57] F. Popp, P. Slowik, E. Eggenhofer et al., "No contribution of multipotent mesenchymal stromal cells to liver regeneration in a rat model of prolonged hepatic injury," Stem Cells, vol. 25, no. 3, pp. 639-645, 2007.

[58] M. E. Quintanilla, F. Ezquer, P. Morales et al., "Intranasal mesenchymal stem cell secretome administration markedly inhibits alcohol and nicotine self-administration and blocks relapse-intake: mechanism and translational options," Stem Cell Research \& Therapy, vol. 10, no. 1, p. 205, 2019. 